\title{
INTERTWINING CERTAIN FRACTIONAL DERIVATIVES
}

\author{
PIERRE PATIE AND THOMAS SIMON
}

\begin{abstract}
We obtain an intertwining relation between some Riemann-Liouville operators of order $\alpha \in(1,2)$, connecting through a certain multiplicative identity in law the one-dimensional marginals of reflected completely asymmetric $\alpha$-stable Lévy processes. An alternative approach based on recurrent extensions of positive self-similar Markov processes and exponential functionals of Lévy processes is also discussed.
\end{abstract}

\section{Introduction AND StATEMEnt of the RESUlt}

Consider for every $\alpha \in(1,2)$ the following operators acting on functions from $\mathbb{R}^{+}$to $\mathbb{R}$ :

$$
D_{+}^{\alpha-1} f(x)=\frac{\mathrm{d}}{\mathrm{d} x} \int_{0}^{x} \frac{f(t)(x-t)^{1-\alpha}}{\Gamma(2-\alpha)} d t \quad \text { and } \quad D_{-}^{\alpha} f(x)=\frac{\mathrm{d}^{2}}{\mathrm{~d} x^{2}} \int_{x}^{\infty} \frac{f(t)(t-x)^{1-\alpha}}{\Gamma(2-\alpha)} d t
$$

for every $x>0$. The operator $D_{+}^{\alpha-1}$ is known as the left-sided Riemann-Liouville (RL) derivative of index $\alpha-1$ and $D_{-}^{\alpha}$ as the right-sided RL derivative of index $\alpha$. Recently, RL derivatives have appeared quite often in various domains of analysis and probability and we refer to Chapter 2 in [14 for a detailed account on them, as well as on other fractional operators. From the analytical viewpoint, RL derivatives extend in a non-local fashion the derivatives of integer order - see (2.2.5) in [14. To name but one classical example of the occurence of RL derivatives in probability, recall that

$$
D_{-}^{\alpha}=D^{2} \circ U^{2-\alpha}
$$

where $D$ is the usual derivative and $U^{2-\alpha}$ the potential associated to the standard $(2-\alpha)$-stable subordinator - see e.g. Exercise (1.6) in [4]. Consider now the operator

$$
\Delta_{+}^{\alpha}=D_{+}^{\alpha-1} \circ D
$$

noticing first that it differs from $D \circ D_{+}^{\alpha-1}=D_{+}^{\alpha}$ - see (2.2.29) in [14] for this latter equality where $D_{+}^{\alpha}$ is the left-sided RL derivative of index $\alpha$ which is analogously defined by

$$
D_{+}^{\alpha} f(x)=\frac{\mathrm{d}^{2}}{\mathrm{~d} x^{2}} \int_{0}^{x} \frac{f(t)(x-t)^{1-\alpha}}{\Gamma(2-\alpha)} d t .
$$

Indeed, an integration by parts shows that for every $x>0$

$$
D_{+}^{\alpha} f(x)=\Delta_{+}^{\alpha} f(x)+\frac{x^{-\alpha}}{\Gamma(1-\alpha)} f(0) .
$$

2000 Mathematics Subject Classification. 60G18, 60G51, 60J25, 26A33, 33E12.

Key words and phrases. Intertwining - Recurrent extension - Reflected process - Riemann-Liouville fractional derivative - Stable Lévy process. 
Another fractional operator related to $\Delta_{+}^{\alpha}$ is the so-called Caputo $\alpha$-fractional derivative which is given by

$$
{ }^{C} D_{+}^{\alpha} f(x)=\frac{1}{\Gamma(2-\alpha)} \int_{0}^{x} f^{\prime \prime}(u)(x-u)^{1-\alpha} d u
$$

see section 2.4 in [14]. A similar integration by parts shows namely that

$$
\Delta_{+}^{\alpha} f(x)={ }^{C} D_{+}^{\alpha} f(x)+\frac{x^{1-\alpha}}{\Gamma(2-\alpha)} f^{\prime}(0) .
$$

For any $\alpha \in(1,2)$, let $\mathcal{V}_{\alpha}$ be the multiplicative kernel acting on functions from $\mathbb{R}^{+}$to $\mathbb{R}$ by

$$
\mathcal{V}_{\alpha} f(x)=\mathbb{E}\left[f\left(x V_{\alpha}\right)\right],
$$

where $V_{\alpha}$ is a positive random variable having the density

$$
v_{\alpha}(t)=\frac{(-\sin \pi \alpha) t^{\alpha-2}(1+t)}{\pi\left(t^{2 \alpha}-2 t^{\alpha} \cos \alpha \pi+1\right)}
$$

- it will be checked soon afterwards that $v_{\alpha}$ is indeed a density function on $\mathbb{R}^{+}$. Setting $\mathcal{C}_{b}^{2}$ for the set of twice continuously differentiable functions $\mathbb{R}^{+} \rightarrow \mathbb{R}$ such that $f^{\prime}$ and $f^{\prime \prime}$ are bounded, consider finally the following domain

$$
\left.\mathcal{D}=\left\{f \in \mathcal{C}_{b}^{2} \text { such that } f^{\prime}(0)=0 \text { and } \exists \gamma>2-\alpha / \lim _{x \rightarrow+\infty} x^{\gamma}\left(|f(x)|+\mid f^{\prime \prime}(x)\right) \mid\right)=0\right\} .
$$

Observe that if $f \in \mathcal{D}$, then necessarily $\exists \gamma>2-\alpha / \lim _{x \rightarrow+\infty} x^{\gamma}\left|f^{\prime}(x)\right|=0$ as well, which can be checked from the decomposition

$$
f(x+1)=f(x)+f^{\prime}(x)+\int_{x}^{x+1}(x+1-y) f^{\prime \prime}(y) d y .
$$

It is also easily seen that $D_{-}^{\alpha}$ and $\mathcal{V}_{\alpha}$ are well-defined on $\mathcal{D}$, and it will be proved in the next section that $\Delta_{+}^{\alpha}$ is well-defined on $\mathcal{V}_{\alpha}(\mathcal{D})$. Our main result is the following intertwining relation between $\Delta_{+}^{\alpha}$ and $D_{-}^{\alpha}$ :

Theorem. For any $f \in \mathcal{D}$, one has

$$
\Delta_{+}^{\alpha} \mathcal{V}_{\alpha} f=\mathcal{V}_{\alpha} D_{-}^{\alpha} f
$$

Intertwining relations between Markov processes have some history and we refer to [7, 12] for a probabilistic account, as well as various examples and applications. See also [18 for a particular analytical study. Though expressed in analytical terms, our result falls within the Markovian framework. It was namely observed in [1] that $\Delta_{+}^{\alpha}\left(\right.$ resp. $\left.D_{-}^{\alpha}\right)$ is the infinitesimal generator of the spectrally positive (resp. spectrally negative) $\alpha$-stable Lévy process reflected at its running supremum. It might be interesting to mention that although the underlying stable Lévy processes are in classical duality, this is no more the case for the reflected processes, so that our result can be viewed as a kind of intertwisted duality relationship for the latter.

The proof of the theorem hinges upon the well-known criterion given in the Proposition 3.2 of [6] and an identity in law connecting the running suprema of completely asymmetric stable processes which was recently obtained in [28], involving the positive variable $T_{\alpha}$ with density

$$
\frac{(-\sin \pi \alpha)\left(1+t^{1 / \alpha}\right)}{\pi \alpha\left(t^{2}-2 t \cos \alpha \pi+1\right)} \text {. }
$$


Notice in passing that our above function $v_{\alpha}$ is the density of the variable $T_{\alpha}^{-1 / \alpha}$, hence it is a density function. Some particular attention is paid to the functional domain upon which the intertwining relation holds and the set $\mathcal{D}$, which we borrowed from [1, appears to be a reasonable and not too small candidate. With the help of some Suprun-type formulæ for the resolvent of spectrally one-sided Lévy processes which had been derived in [23], we can also identify the Fellerian domains of $\Delta_{+}^{\alpha}$ and $D_{-}^{\alpha}$. In theory, those Fellerian domains yield an optimal formulation for the theorem, although they do not seem very tractable.

In Section 3 we discuss another approach which consists in interpreting the stable process reflected at its infimum as the unique self-similar recurrent extension leaving 0 continuously of the stable process killed when it enters the negative half-line. This identification had been roughly explained in Example 3 of [25] and here we can also check analytically that these two Feller processes have the same infinitesimal generators. This gives another proof, looking somewhat more unified, of all the results contained in the Appendix in [1]. An independent proof of the identity in law between suprema of completely asymmetric stable processes, which is the keyargument for the theorem, is also proposed, involving some closed formulæ for the exponential functionals of certain Lévy processes which had been established in [21, 22]. Though overall a bit lenghtier, we believe that this second point of view provides some unity to our interweaving relationship, which appears to be coherent with several apparently disconnected identities.

To conclude this introduction, we stress that the positive random variable $Z_{\beta}$ with density

$$
\frac{(-\sin \pi \alpha)}{\pi(\alpha-1)\left(t^{2}-2 t \cos \alpha \pi+1\right)}=\frac{\sin \pi \beta}{\pi \beta\left(t^{2}+2 t \cos \beta \pi+1\right)}
$$

where $\beta=\alpha-1 \in(0,1)$, which can be viewed as a cut off Cauchy variable, has already occured in several distinct areas of the literature, especially through its power transforms $Y_{\beta}=Z_{\beta}^{1 / \beta}$. See for instance Formula XI.11.6 in [30] for connections with the $\beta$-fractional power of linear operators, Theorem 1.1 and Theorem 1.2 in [15] for mixture representations of the Linnik and Mittag-Leffler distributions of index $\beta$, and more general geometric stable distributions, or Exercise 4.21 (3) in [8] which shows that $Y_{\beta}$ has the same law as the independent quotient of two standard positive $\beta$-stable laws. The above variables $V_{\alpha}$ and $T_{\alpha}$ are less classical than $Z_{\beta}$ but one may of course wonder if they are not particular instances of a family of positive variables connecting suprema of general stable processes in duality, or a broader class of fractional operators than the one we consider in the present article. We plan to tackle this question in some further research.

\section{Proof of the theOREM}

Let $(Z, \mathbb{P})$ be a spectrally negative stable Lévy process of index $\alpha \in(1,2)$ starting at 0 , with Lévy density

$$
\nu_{\alpha}(y)=\frac{|y|^{-(\alpha+1)}}{\Gamma(-\alpha)} \mathbf{1}_{\{y<0\}},
$$

so that the Lévy-Khintchine formula reads

$$
\mathbb{E}\left[e^{\lambda Z_{t}}\right]=e^{t \lambda^{\alpha}}
$$

for every $t, \lambda \geq 0$. See e.g. Chapters VII \& VIII in [2] for an account on completely asymmetric stable processes. Setting $S_{t}=\sup \left\{Z_{s}, s \leq t\right\}$ for the associated running supremum and introducing

$$
T_{x}=\inf \left\{t>0, Z_{t}=x\right\}=\inf \left\{t>0, S_{t}=x\right\}
$$


recall that $T_{1} \stackrel{\mathrm{d}}{=} S_{1}^{-\alpha}$ is a standard positive $(1 / \alpha)$-stable law, viz.

$$
\mathbb{E}\left[e^{\lambda T_{1}}\right]=e^{-\lambda^{1 / \alpha}}
$$

for every $\lambda \geq 0$. Denoting by $I_{t}=\inf \left\{Z_{s}, s \leq t\right\}$ the running infimum, consider the reflected processes

$$
X_{t}=S_{t}-Z_{t} \text { and } \hat{X}_{t}=Z_{t}-I_{t} .
$$

Notice that if $\hat{Z}=-Z$ is the dual process and if $Y_{t}=\hat{S}_{t}-\hat{Z}_{t}$ and $\hat{Y}_{t}=\hat{Z}_{t}-\hat{I}_{t}$ are the corresponding reflected processes, then $Y=\hat{X}$ and $\hat{Y}=X$. It is a basic fact from fluctuation theory - see e.g. Proposition VI.1 in [2] - that $X$ and $\hat{X}$ are Feller processes and we will denote by $\mathbb{P}_{x}$ resp. $\hat{\mathbb{P}}_{x}$ their laws starting from $x \geq 0$. The infinitesimal generators $L$ and $\hat{L}$ of $X$ and $\hat{X}$ have recently been expressed in [1], in three different forms. Recalling that by definition

$$
L f(x)=\lim _{t \rightarrow 0} \frac{\mathbb{E}_{x}\left[f\left(X_{t}\right)\right]-f(x)}{t} \quad \text { resp. } \quad \hat{L} f(x)=\lim _{t \rightarrow 0} \frac{\hat{\mathbb{E}}_{x}\left[f\left(\hat{X}_{t}\right)\right]-f(x)}{t}
$$

for every continuous function $f: \mathbb{R}^{+} \rightarrow \mathbb{R}$ such that the limit in the right-hand side of the first (resp. second) equality exists uniformly, let us denote by Dom $L$ (resp. Dom $\hat{L}$ ) the set of such functions. Choosing then Riemann Liouville's form in Proposition A.1 of [1], one has $\mathcal{D} \subset$ Dom $L$ and

$$
L f(x)=D_{-}^{\alpha} f(x), \quad x>0
$$

for every $f \in \mathcal{D}$. Besides, one has

$$
\hat{L} f(x)=\Delta_{+}^{\alpha} f(x), \quad x>0
$$

for every $f \in \mathcal{C}_{b}^{2}$ such that $f^{\prime}(0)=0$. The next proposition shows that $\Delta_{+}^{\alpha}$ is well-defined on $\mathcal{V}_{\alpha}(\mathcal{D})$ so that the statement of our theorem makes sense:

Proposition 2.1. One has $\mathcal{V}_{\alpha}(\mathcal{D}) \subset$ Dom $\hat{L}$.

Proof. Since $v_{\alpha}(t)$ is of order $t^{-(1+\alpha)}$, one sees by dominated convergence that for any $f \in \mathcal{D}$, the function $\mathcal{V}_{\alpha} f$ is continuously differentiable on $\mathbb{R}^{+}$with bounded derivative

$$
\left(\mathcal{V}_{\alpha} f\right)^{\prime}(x)=\mathbb{E}\left[V_{\alpha} f^{\prime}\left(x V_{\alpha}\right)\right]
$$

- whence in particular $\left(\mathcal{V}_{\alpha} f\right)^{\prime}(0)=0$, and twice continuously differentiable on $(0,+\infty)$ with second derivative

$$
\left(\mathcal{V}_{\alpha} f\right)^{\prime \prime}(x)=\mathbb{E}\left[V_{\alpha}^{2} f^{\prime \prime}\left(x V_{\alpha}\right)\right], \quad x>0 .
$$

On the other hand, the right-hand side in the above equality might not be bounded when $x \rightarrow 0$ because $\mathbb{E}\left[V_{\alpha}^{2}\right]=+\infty$. An easy change of variable shows however that for every $f \in \mathcal{D}$, the quantity

$$
\left(x^{\gamma} \wedge 1\right) \int_{0}^{\infty}\left(t^{1-\alpha} \wedge 1\right) f^{\prime \prime}(x t) d t
$$

remains bounded on $(0,+\infty)$ for some $\gamma<2-\alpha$, so that $\left(x^{\gamma} \wedge 1\right)\left(\mathcal{V}_{\alpha} f\right)^{\prime \prime}(x)$ is bounded on $(0,+\infty)$. Hence, we need to show that in Proposition 4 of [1], the global boundedness assumption on $F^{\prime \prime}$ can be relaxed into $\left(x^{\gamma} \wedge 1\right) F^{\prime \prime}(x)$ bounded on $(0,+\infty)$ for some $\gamma \in[0,2-\alpha)$. A persual of the proof shows that it is indeed the case: the relaxed condition changes namely the right-hand side of the second inequality in (4.11) therein into

$$
C_{\alpha} \mathbf{E}_{y}\left[\int_{0}^{t}\left(Y_{s}^{2-\alpha}+Y_{s}^{2-\alpha-\gamma}\right) d s\right]
$$


for some positive finite constant $C_{\alpha}$, where $\mathbf{P}_{y}$ stands for the law of $Y$ (with their notation for $Y$, which matches ours) starting at $y \geq 0$. Using self-similarity, the expectation is then bounded by

$$
C_{\alpha}^{\prime}\left(\mathbf{E}_{y}\left[Y_{1}^{2-\alpha}\right]+\mathbf{E}_{y}\left[Y_{1}^{2-\alpha-\gamma}\right]\right) \leq C_{\alpha, \gamma} \mathbf{E}_{y}\left[Y_{1}^{2-\alpha}\right]
$$

for some other positive finite constants $C_{\alpha}^{\prime}, C_{\alpha, \gamma}$. Notice now by the Feller property and the identification $Y=\hat{X}$ that the law of $Y$ under $\mathbf{P}_{y}$ is that of the process $\left\{Z_{t}+\left(y \vee\left(-I_{t}\right)\right), t \geq 0\right\}$ under $\mathbb{P}$. Since $0<2-\alpha<1<\alpha$, one has e.g. from Proposition VIII.4 in [2] and the comment thereafter

$$
\mathbf{E}_{y}\left[Y_{1}^{2-\alpha}\right]=\mathbb{E}\left[S_{1}^{2-\alpha}\right]+\mathbb{E}\left[\left(y \vee\left(-I_{1}\right)\right)^{2-\alpha}\right]<+\infty,
$$

which was already used in [1. All of this shows that (4.12) remains unchanged for $F$ under the relaxed condition, which entails exactly as in [1 that the limit in the right-hand side of (2.2) exists pointwise for any $f \in \mathcal{V}_{\alpha}(\mathcal{D})$. To finish the proof, notice first by the Feller property that the transition densities of $\hat{X}$ form a strongly continuous contraction semigroup on the Banach space of continuous functions $\mathbb{R}^{+} \rightarrow \mathbb{R}$ tending to 0 at infinity, to which belongs $\mathcal{V}_{\alpha}(\mathcal{D})$. Hence, one can apply the whole semigroup theory recalled e.g. in Section 31 of [26]. In particular, a result of K. Itô - see Lemma 31.7 in [26] shows that the limit in the right-hand side of (2.2) is actually uniform for any $f \in \mathcal{V}_{\alpha}(\mathcal{D})$, whence $f \in \operatorname{Dom} \hat{L}$ as desired.

Let us now consider the Fellerian domains of $L$ and $\hat{L}$. Setting $\mathcal{C}_{0}$ for the set of continuous functions $\mathbb{R}^{+} \rightarrow \mathbb{R}$ tending to 0 at infinity, the Feller property states that both functions

$$
x \mapsto \mathbb{E}_{x}\left[f\left(X_{t}\right)\right] \text { and } x \mapsto \hat{\mathbb{E}}_{x}\left[f\left(\hat{X}_{t}\right)\right]
$$

are in $\mathcal{C}_{0}$ whenever $f \in \mathcal{C}_{0}$. The Fellerian domains of $L$ and $\hat{L}$, which we denote respectively by $\mathcal{D}(L)$ and $\mathcal{D}(\hat{L})$ are made out of those functions in $\mathcal{C}_{0}$ such that the limits in (2.2) exist uniformly - see e.g. Definition VII (1.1) in [24]. It is well-known from semigroup theory - see e.g. Proposition VII (1.4) in [24] - that

$$
\mathcal{D}(L)=U_{q}\left(\mathcal{C}_{0}\right) \text { and } \mathcal{D}(\hat{L})=\hat{U}_{q}\left(\mathcal{C}_{0}\right)
$$

for every $q>0$, where $U_{q}, \hat{U}_{q}$ are the resolvent operators defined by

$$
U_{q} f(x)=\int_{0}^{\infty} e^{-q t} \mathbb{E}_{x}\left[f\left(X_{t}\right)\right] d t \quad \text { and } \quad \hat{U}_{q} f(x)=\int_{0}^{\infty} e^{-q t} \hat{\mathbb{E}}_{x}\left[f\left(\hat{X}_{t}\right)\right] d t .
$$

It follows from the definitions that

$$
\operatorname{Dom} L \cap \mathcal{C}_{0}=\mathcal{D}(L) \text { and } \operatorname{Dom} \hat{L} \cap \mathcal{C}_{0}=\mathcal{D}(\hat{L}) .
$$

The next proposition gives a full description of $\mathcal{D}(L)$ and $\mathcal{D}(\hat{L})$ in terms of the functions $F_{\alpha}(x)=$ $E_{\alpha}\left(x^{\alpha}\right)$ and its derivatives, where $E_{\alpha}$ is the Mittag-Leffler function of index $\alpha$ which is defined by

$$
E_{\alpha}(x)=\sum_{n=0}^{\infty} \frac{x^{n}}{\Gamma(\alpha n+1)} .
$$

For every $f \in \mathcal{C}_{0}$, introduce the further notation

$$
\lambda_{f}=\int_{0}^{\infty} e^{-y} f(y) d y .
$$


Proposition 2.2. One has $\mathcal{D}(\hat{L})=\left\{x \mapsto \lambda_{f} F_{\alpha}(x)-F_{\alpha}^{\prime} \star f(x), \quad f \in \mathcal{C}_{0}\right\}$ and

$$
\mathcal{D}(L)=\left\{x \mapsto e^{-x} \int_{0}^{x} F_{\alpha}^{\prime \prime}(y) f(y) d y+\int_{x}^{\infty}\left(e^{-x} F_{\alpha}^{\prime \prime}(y)-F_{\alpha}^{\prime}(y-x)\right) f(y) d y, \quad f \in \mathcal{C}_{0}\right\} .
$$

Proof. Let us start with $\mathcal{D}(\hat{L})=\hat{U}^{1}\left(\mathcal{C}_{0}\right)$. The resolvent density of $\hat{X}=Y$ killed when entering the half-line $(a, \infty)$ has been computed for every $a>0$ in [23], Theorem 1 (i). Notice first that the notations therein yield $Z^{(1)}(x)=F_{\alpha}(x)$ and $W^{(1)}(x)=F_{\alpha}^{\prime}(x)$. Besides, it was proved in Theorem 1 of [28] that the function $F_{\alpha}-F_{\alpha}^{\prime}$ is completely monotone, so that in particular $F_{\alpha}(x)-F_{\alpha}^{\prime}(x) \rightarrow 0$ as $x \rightarrow+\infty$ which, together with Formula 18.1 (10) in [9], entails

$$
\frac{W^{(1)}(a-y)}{Z^{(1)}(a)} \rightarrow e^{-y} \quad \text { as } a \rightarrow+\infty
$$

for every $y \geq 0$. By monotone convergence, letting $a \rightarrow+\infty$ shows that the density of $\hat{U}^{1}$ is

$$
\hat{u}^{1}(x, y)=e^{-y} F_{\alpha}(x)-F_{\alpha}^{\prime}(x-y) \mathbf{1}_{\{y \leq x\}},
$$

whence

$$
\hat{U}^{1} f(x)=\left(\int_{0}^{\infty} e^{-y} f(y) d y\right) F_{\alpha}(x)-\int_{0}^{x} F_{\alpha}^{\prime}(x-y) f(y) d y=\lambda_{f} F_{\alpha}(x)-F_{\alpha}^{\prime} \star f(x)
$$

for every $f \in \mathcal{C}_{0}$ and $x \geq 0$, which is the required identification for $\mathcal{D}(\hat{L})$.

Before identifying $\mathcal{D}(L)=\hat{U}^{1}\left(\mathcal{C}_{0}\right)$, let us check that the two integrals in the definition are well-defined for every $f \in \mathcal{C}_{0}$ and $x \geq 0$ : the convergence of the proper integral comes from the easily shown behaviour $F_{\alpha}^{\prime \prime}(y) \sim(\alpha-1) y^{\alpha-2} / \Gamma(2 \alpha)$ as $y \rightarrow 0$, and the existence of the improper one is proved in combining several times formulæ (6) and (43) in [11], which yield

$$
\alpha F_{\alpha}^{\prime}(z)=e^{z}+\mathrm{O}\left(z^{-(1+\alpha)}\right) \text { and } \alpha F_{\alpha}^{\prime \prime}(z)=e^{z}+\mathrm{O}\left(z^{-(2+\alpha)}\right)
$$

as $z \rightarrow+\infty$. The latter asymptotics also entail, with the notations of [23],

$$
\frac{W^{(1)}(a-x)}{W_{+}^{(1)^{\prime}}(a)}=\frac{F_{\alpha}^{\prime}(a-x)}{F_{\alpha}^{\prime \prime}(a)} \rightarrow e^{-x} \quad \text { as } a \rightarrow+\infty .
$$

Since moreover $W^{(1)}(0)=F_{\alpha}^{\prime}(0)=0$, one obtains from Theorem 1 (ii) in [23] and monotone convergence the following expression for the density of $U_{1}$ :

$$
u^{1}(x, y)=e^{-x} F_{\alpha}^{\prime \prime}(y)-F_{\alpha}^{\prime}(y-x) \mathbf{1}_{\{y \geq x\}}
$$

whence, as above, the desired expression for $\mathcal{D}(\hat{L})$.

Remarks 2.3. (a) If $g=\hat{U}^{1} f \in \mathcal{D}(\hat{L})$, then $g(0)=\lambda_{f}$ and $g$ is continuously differentiable on $\mathbb{R}^{+}$with derivative $g^{\prime}(x)=\lambda_{f} F_{\alpha}^{\prime}(x)-F_{\alpha}^{\prime \prime} \star f(x)$, so that in particular $g^{\prime}(0)=0$. On the other hand, choosing $f$ non differentiable shows that $\mathcal{D}(L)$ contains functions which are not $\mathcal{C}^{2}$, as of course might be expected from the expression of $L$. See Chapter 2 in [14] for more material concerning the domains of Riemann-Liouville derivatives.

(b) If $g=U^{1} f \in \mathcal{D}(L)$, then

$$
g(0)=\int_{0}^{\infty}\left(F_{\alpha}^{\prime \prime}(y)-F_{\alpha}^{\prime}(y)\right) f(y) d y
$$


and $g$ is continuously differentiable on $\mathbb{R}^{+}$with derivative

$$
g^{\prime}(x)=\int_{x}^{\infty}\left(F_{\alpha}^{\prime \prime}(y-x)-e^{-x} F_{\alpha}^{\prime \prime}(y)\right) f(y) d y-e^{-x} \int_{0}^{x} F_{\alpha}^{\prime \prime}(y) d y,
$$

so that in particular $g^{\prime}(0)=0$. Again, $\mathcal{D}(\hat{L})$ contains functions which are not $\mathcal{C}^{2}$.

End of the proof. We will use the inclusion $(2) \Rightarrow(3)$ in the Proposition 3.2 of [6]. We already know that $X$ and $\hat{X}$ are Feller processes and it follows easily from the $(1 / \alpha)$-self-similarity of $Z$ that they are also $(1 / \alpha)$-self-similar Markov processes. More precisely, one has

$$
\left(X^{b}, \mathbb{P}_{x}\right) \stackrel{\mathrm{d}}{=}\left(X, \mathbb{P}_{b x}\right) \quad \text { and } \quad\left(\hat{X}^{b}, \hat{\mathbb{P}}_{x}\right) \stackrel{\mathrm{d}}{=}\left(\hat{X}, \hat{\mathbb{P}}_{b x}\right)
$$

for every $b>0$, with $X_{t}^{b}=b X_{b^{-\alpha} t}$ and an analogous notation for $\hat{X}^{b}$. Since $\alpha \neq 1$, this shows that they are not semi-stable Markov processes viz. 1-self-similar Markov processes - see (1.b) in [6]. However, a perusal of the proof of Proposition 3.2 in [6] shows that its statement remains unchanged when considering $(1 / \alpha)$-self-similar Markov processes for every $\alpha>0$ and not just $\alpha=1$.

We next show that the distribution of $\hat{X}$ is determinate under $\hat{\mathbb{P}}_{0}$, with the notation of [6]. From e.g. Proposition VI.3 in [2], the law of $\hat{X}_{t}$ under $\hat{\mathbb{P}}_{0}$ is that of $S_{t}$ under $\mathbb{P}$. Hence, taking the Laplace transforms, we need to show that if $f, g \in \mathcal{C}_{0}$ are such that

$$
\mathbb{E}\left[\int_{0}^{\infty} e^{-\lambda t} f\left(S_{t}\right) d t\right]=\mathbb{E}\left[\int_{0}^{\infty} e^{-\lambda t} g\left(S_{t}\right) d t\right]
$$

for every $\lambda>0$, then $f=g$. The latter is a basic property of $(1 / \alpha)$-stable subordinators but we will give some details for the reader's comfort. Recalling the notation $T_{x}=\inf \left\{t>0, Z_{t}=x\right\}$ for every $x \geq 0$, we know that $\left\{T_{x}, x \geq 0\right\}$ is a standard $(1 / \alpha)$-stable subordinator since $Z$ has no positive jumps. Besides, one has Leb $\left(\mathbb{R}^{+}-\cup_{x \geq 0}\left(T_{x-}, T_{x}\right)\right)=0$ a.s. - see e.g. the beginning of Section III.5 in [2], so that (2.4) entails

$$
\mathbb{E}\left[\sum_{x>0} f(x) e^{-\lambda T_{x}-}\left(1-e^{-\lambda \Delta T_{x}}\right)\right]=\mathbb{E}\left[\sum_{x>0} g(x) e^{-\lambda T_{x}-}\left(1-e^{-\lambda \Delta T_{x}}\right)\right] .
$$

The so-called Master's Formula - see e.g. Proposition XII (1.10) in [24] - yields then

$$
\mathbb{E}\left[\int_{0}^{\infty} f(x) e^{-\lambda T_{x}-}\left(\int_{0}^{\infty} \frac{1-e^{-\lambda u}}{u^{\alpha+1}} d u\right) d x\right]=\mathbb{E}\left[\int_{0}^{\infty} g(x) e^{-\lambda T_{x-}}\left(\int_{0}^{\infty} \frac{1-e^{-\lambda u}}{u^{\alpha+1}} d u\right) d x\right],
$$

whence

$$
\int_{0}^{\infty} f(x) e^{-\lambda^{1 / \alpha} x} d x=\int_{0}^{\infty} g(x) e^{-\lambda^{1 / \alpha} x} d x
$$

and the required identification $f=g$ by inversion of the Laplace transforms.

Last, we see from Proposition VI. 3 in [2] and Formula (9) in [28] (beware the inverse notations) that

$$
X_{1} \stackrel{\mathrm{d}}{=} \hat{S}_{1} \stackrel{\mathrm{d}}{=} T_{\alpha}^{-1 / \alpha} \times S_{1} \stackrel{\mathrm{d}}{=} V_{\alpha} \times S_{1} \stackrel{\mathrm{d}}{=} V_{\alpha} \times \hat{X}_{1},
$$

where the identity $T_{\alpha}^{-1 / \alpha} \stackrel{\mathrm{d}}{=} V_{\alpha}$ was mentioned in the introduction and follows from a change of variable. Putting everything together, Proposition 3.2 in [6] shows that $\Delta_{+}^{\alpha} \mathcal{V}_{\alpha} f=\mathcal{V}_{\alpha} D_{-}^{\alpha} f$ for every $f \in \mathcal{D}(L)$ such that $\mathcal{V}_{\alpha} f \in \mathcal{D}(\hat{L})$. Supposing now that $f \in \mathcal{D} \subset \operatorname{Dom} L \cap \mathcal{C}_{0}=\mathcal{D}(L)$, 
Proposition 2.1 shows that $\mathcal{V}_{\alpha} f \in$ Dom $\hat{L}$ and it follows immediately from dominated convergence that $\mathcal{V}_{\alpha} f \in \mathcal{C}_{0}$. Hence, $\mathcal{V}_{\alpha} f \in \operatorname{Dom} \hat{L} \cap \mathcal{C}_{0}=\mathcal{D}(\hat{L})$ and we have shown

$$
\Delta_{+}^{\alpha} \mathcal{V}_{\alpha} f=\mathcal{V}_{\alpha} D_{-}^{\alpha} f
$$

for every $f \in \mathcal{D}$, as required.

Remarks 2.4. (a) The interweaving relationship

$$
{ }^{C} D_{+}^{\alpha} \mathcal{V}_{\alpha} f=\mathcal{V}_{\alpha} D_{-}^{\alpha} f
$$

also holds over $\mathcal{D}$ because of the identity (1.2). Actually, since $f^{\prime}(0)=0$ for every $f \in \mathcal{D}(\hat{L})$, the operator ${ }^{C} D_{+}^{\alpha}$ coincides with the generator $\hat{L}$ of $\hat{X}$.

(b) As mentioned during the proof, Proposition 3.2 in [6] shows that

$$
\Delta_{+}^{\alpha} \mathcal{V}_{\alpha} f=\mathcal{V}_{\alpha} D_{-}^{\alpha} f
$$

holds for every $f \in \mathcal{D}(L)$ such that $\mathcal{V}_{\alpha} f \in \mathcal{D}(\hat{L})$. The domains $\mathcal{D}(L)$ and $\mathcal{D}(\hat{L})$ have been identified in Proposition 2.2 and allow rougher functions than $\mathcal{D}$ but their formulations are unfortunately not very tractable, contrary to $\mathcal{D}$.

We close this section with an interesting complete monotonicity property for the MittagLeffler function $E_{\alpha}$. The latter is actually a direct consequence of Proposition 2 in [23], but we present here a proof based on generators which is perhaps more transparent. Recall that a smooth function $f:] 0,+\infty\left[\rightarrow \mathbb{R}^{+}\right.$is said to be completely monotone (CM) if

$$
(-1)^{n} \frac{\mathrm{d}^{n} f}{\mathrm{~d} x^{n}} \geq 0, \quad n \geq 1
$$

By Bernstein's theorem, when $f(x) \rightarrow 1$ as $x \rightarrow 0$ this is equivalent to the fact that $f$ is the Laplace transform of a probability measure over $\mathbb{R}^{+}$. A classical result by Pollard states that for any $\alpha \in(0,1]$ the function $E_{\alpha}(-x)$ is CM, a property which does not hold anymore if $\alpha>1$ - see [27] for a proof of these latter facts and more on this topic. Like $1 / x$, the function $x \mapsto E_{\alpha}(1 / x)$ is CM by positivity of the coefficient in the series expansion - see Section 4 in [17] for further properties as well as general references on complete monotonicity. The following proposition shows a related property in the case $\alpha \in[1,2]$ :

Proposition 2.5. For any $\alpha \in[1,2]$ the function $x \mapsto 1 / E_{\alpha}(x)$ is CM.

Proof. The case $\alpha=1$ is straightforward because $E_{1}(x)=e^{x}$ and the case $\alpha=2$ follows from Lévy's formula: one has

$$
\frac{1}{E_{2}(x)}=\frac{1}{\cosh \sqrt{x}}=\mathbb{E}\left[e^{-x \tau}\right]
$$

with $\tau=\inf \left\{t>0,\left|B_{t}\right|>2^{-1 / 4}\right\}$ and $B$ a standard Brownian motion - see e.g. Exercise II (3.10) in [24], so that $1 / E_{2}(x)$ is CM by Bernstein's theorem. For the remaining case $\alpha \in(1,2)$ we will follow roughly the same arguments as Theorem 2.1 and 2.6 in [20]. Setting

$$
E_{\alpha}^{q}(x)=E_{\alpha}\left(q x^{\alpha}\right) f_{q}(x)
$$

for every $q>0$, where $f_{q}$ is some smooth function such that $f_{q}(x)=1$ for all $x \leq 1$ and $f_{q}(x)=0$ for all $x \geq 2$, say, we see that $E_{\alpha}^{q} \in \mathcal{D}\left(\Delta_{+}^{\alpha}\right)$. On the other hand, it follows from Lemma 2.23 in [14] that

$$
\Delta_{+}^{\alpha} E_{\alpha}^{q}(x)=q E_{\alpha}^{q}(x)
$$


for every $x \leq 1$. From Definition VII (1.8) and Exercise VII (1.24) in [24] - this shows that $t \mapsto e^{-q T_{1}^{+} \wedge t} E_{\alpha}^{q}\left(\hat{X}_{T_{1}^{+} \wedge t}\right)$ is a martingale under $\hat{\mathbb{P}}_{0}$, where

$$
T_{1}^{+}=\inf \left\{s>0, \hat{X}_{s} \geq 1\right\}=\inf \left\{s>0, \hat{X}_{s}=1\right\}
$$

(recall that $\hat{X}$ has no positive jumps for the second equality). The optional sampling theorem entails

$$
1=\hat{\mathbb{E}}_{0}\left[e^{-q T_{1}^{+} \wedge t} E_{\alpha}^{q}\left(\hat{X}_{T_{1}^{+} \wedge t}\right)\right] \rightarrow \hat{\mathbb{E}}_{0}\left[e^{-q T_{1}^{+}} E_{\alpha}^{q}\left(\hat{X}_{T_{1}^{+}}\right)\right]=E_{\alpha}(q) \hat{\mathbb{E}}_{0}\left[e^{-q T_{1}^{+}}\right]
$$

as $t \rightarrow \infty$, by dominated convergence because $\hat{X}_{T_{1}^{+} \wedge t} \in[0,1]$ a.s. This completes the proof by Bernstein's theorem.

Remark 2.6. It is plain from self-similarity, the Markov property and the absence of positive jumps that the variable $T_{1}^{+}$is self-decomposable. By a well-known argument - see the proof of Proposition 2.6 in [20] for details - this shows that the mapping

$$
x \mapsto \exp -\left[x E_{\alpha}^{\prime}(x) / E_{\alpha}(x)\right]
$$

is also CM as the Laplace transform of a positive infinitely divisible random variable.

\section{Another APproach With ReCURREnt eXtensions}

The purpose of this section is two-fold. First, we will give another proof of the identifications $L=D_{-}^{\alpha}$ and $\hat{L}=\Delta_{+}^{\alpha}$, viewing the reflected process $X$ (resp. $\hat{X}$ ) as a recurrent extension of the process $\hat{Z}$ (resp. $Z$ ) killed when entering the negative half-line, and using the classical expression of the infinitesimal generator of the unkilled stable Lévy process. Actually the approach works for every strictly stable process $Z$ such that $|Z|$ is not a subordinator, making it possible to retrieve the whole Appendix of [1]. Second, we will derive a proof of the identity

$$
X_{1} \stackrel{\mathrm{d}}{=} V_{\alpha} \times \hat{X}_{1},
$$

which is independent of [28] and relies upon closed expressions for the densities of exponential functional of certain spectrally negative Lévy processes that had been carried out in [21, 22].

3.1. Retrieving the generators of reflected stable processes. Let $\left(Z, \mathbb{Q}_{x}\right)$ be a strictly stable Lévy process of index $\alpha \in(0,2)$ such that $|Z|$ is not a subordinator, starting from $x \in \mathbb{R}$. We refer e.g. to Chapter VIII in [2] for details. The density of the Lévy measure is

$$
\nu(y)=c_{+} y^{-\alpha-1} \mathbf{1}_{\{y>0\}}+c_{-}|y|^{-\alpha-1} \mathbf{1}_{\{y<0\}},
$$

where $c_{+}, c_{-}$are nonnegative constants such that $c_{+}+c_{-}>0$. When $\alpha=1$ we suppose that $Z$ is a symmetric Cauchy process viz. $c_{+}=c_{-}=c>0$. Again, we will use the notations $S_{t}=\sup \left\{Z_{s}, s \leq t\right\}, I_{t}=\inf \left\{Z_{s}, s \leq t\right\}, X_{t}=S_{t}-Z_{t}$ and $\hat{X}_{t}=Z_{t}-I_{t}$. Setting also $T=$ $\inf \left\{t>0, Z_{t} \leq 0\right\}$, consider now the killed process

$$
R_{t}=Z_{t} \mathbf{1}_{\{T>t\}}
$$

Again, since $\left(T, \mathbb{Q}_{x}\right) \stackrel{\mathrm{d}}{=}\left(x^{\alpha} T, \mathbb{Q}_{1}\right)$ for every $x \geq 0$, one sees that $\left(R, \mathbb{Q}_{x}\right)$ is a positive $(1 / \alpha)$-selfsimilar Markov processes, viz. a Feller process taking values in $\mathbb{R}^{+}$(here, with 0 as an absorbing state) and fulfilling the scaling property

$$
\left(R^{b}, \mathbb{Q}_{x}\right) \stackrel{\mathrm{d}}{=}\left(R, \mathbb{Q}_{b x}\right)
$$


for every $b>0$, with the notation $R_{t}^{b}=b R_{b^{-\alpha} t}$. As noticed in Example 3 of [25], the reflected process $\left(\hat{X}, \hat{\mathbb{P}}_{x}\right)$ (with $\hat{\mathbb{P}}_{x}$ defined analogously as in the preceding section) can be viewed as the unique self-similar recurrent extension of $\left(R, \mathbb{Q}_{x}\right)$ leaving 0 continuously. Roughly speaking, for every $x>0$ those two processes have the same law until the a.s. finite time $T$ resp. $\hat{T}=\inf \left\{t>0, \hat{X}_{t} \leq 0\right\}$ but 0 is a regular boundary point for $\hat{X}$, which is left instantaneously and continuously. The Feller process $\hat{X}$ has also infinite lifetime. See [10, 25] for precise accounts on recurrent extensions.

We now identify the generator $\hat{\mathcal{R}}$ of the reflected process $\hat{X}$ viewed as a recurrent extension of $R$, retrieving in a unified manner all the results contained in the Appendix of [1]. Beware that since we consider the process reflected at its infimum, our notation is reverse to that of [1]. As in Section 2, we set $\left(\hat{U}_{q}\right)_{q \geq 0}$ for the resolvent of $\hat{X}$. For every $\alpha \in(0,1)$ and $f \in \mathcal{D}$, we use the same notations

$$
D_{-}^{\alpha} f(x)=\frac{\mathrm{d}^{2}}{\mathrm{~d} x^{2}} \int_{x}^{\infty} \frac{f(t)(t-x)^{1-\alpha}}{\Gamma(2-\alpha)} d t=\frac{\mathrm{d}}{\mathrm{d} x} \int_{x}^{\infty} \frac{f(t)(t-x)^{-\alpha}}{\alpha \Gamma(-\alpha)} d t
$$

and

$$
D_{+}^{\alpha} f(x)=\frac{\mathrm{d}^{2}}{\mathrm{~d} x^{2}} \int_{0}^{x} \frac{f(t)(x-t)^{1-\alpha}}{\Gamma(2-\alpha)} d t=-\frac{\mathrm{d}}{\mathrm{d} x} \int_{0}^{x} \frac{f(t)(x-t)^{-\alpha}}{\alpha \Gamma(-\alpha)} d t .
$$

Proposition 3.1 (Bernyk-Dalang-Peskir). For every $f \in \mathcal{D}$ and $x>0$, one has

$$
\hat{\mathcal{R}} f(x)=\Gamma(-\alpha)\left(c_{-} D_{+}^{\alpha} f(x)+c_{+} D_{-}^{\alpha} f(x)\right)+\frac{c_{-} f(0)}{\alpha x^{\alpha}}
$$

if $\alpha \neq 1$, and

$$
\hat{\mathcal{R}} f(x)=c\left(\frac{\mathrm{d}^{2}}{\mathrm{~d} x^{2}} \int_{0}^{\infty} f(t) \log \left(\frac{1}{|x-t|}\right) d t+\frac{f(0)}{x}\right)
$$

if $\alpha=1$.

Proof. Fix $f \in \mathcal{D}$ and $x>0$. For every $M>0$, define $f_{M}$ over $\mathbb{R}$ in setting $f_{M}(x)=f(x)$ for every $x \geq 0, f_{M}(x)=f(0)$ for every $-M<x<0$, and letting $f_{M}(x) \rightarrow 0$ smoothly as $x \rightarrow-\infty$. Then $f_{M} \in \mathcal{C}_{b}^{2}(\mathbb{R})$ except possibly at zero where its left and right second derivatives are bounded, and $f_{M}(x) \rightarrow 0$ as $|x| \rightarrow+\infty$. Besides, with an abuse of notation, one can write $\hat{\mathcal{R}} f(x)=\hat{\mathcal{R}} f_{M}(x)$ for every $M>0$. Introducing the resolvent of $R$

$$
\mathbf{U}_{q} g(x)=\int_{0}^{\infty} e^{-q t} \mathbb{E}_{x}\left[g\left(Z_{t}\right) \mathbf{1}_{\{T>t\}}\right] d t
$$

for every $q, x>0$ and $g: \mathbb{R} \rightarrow \mathbb{R}^{+}$measurable, Theorem 2 (i) in [25] yields

$$
\hat{U}_{q} f_{M}(x)=\mathbf{U}_{q} f_{M}(x)+\mathbb{E}_{x}\left[e^{-q T}\right] \hat{U}_{q} f_{M}(0) .
$$

Recall from semigroup theory - see e.g. Exercise VII (1.15) in [24] - that

$$
\lim _{q \rightarrow \infty} q^{2} \hat{U}_{q} f_{M}(x)-q f_{M}(x)=\hat{\mathcal{R}} f_{M}(x)=\hat{\mathcal{R}} f(x),
$$

which altogether with the notation $\hat{T}_{1}=\inf \left\{t>0, \hat{Z}_{t} \geq 1\right\}$ entails

$$
\begin{aligned}
\hat{\mathcal{R}} f(x) & =\lim _{q \rightarrow \infty}\left(q^{2} \mathbf{U}_{q} f_{M}(x)-q f_{M}(x)+q^{2} \hat{U}_{q} f_{M}(0) \mathbb{E}_{x}\left[e^{-q T}\right]\right) \\
& =\lim _{q \rightarrow \infty}\left(q^{2} \mathbf{U}_{q} f_{M}(x)-q f_{M}(x)+x^{-\alpha} f(0) q \mathbb{E}_{0}\left[e^{-q \hat{T}_{1}}\right]\right),
\end{aligned}
$$


the second equality being a consequence of self-similarity and the easy fact $\lim _{q \rightarrow \infty} q \hat{U}_{q} f_{M}(0)=$ $f_{M}(0)=f(0)$. Proposition VIII.4 in [2], a standard self-similarity argument and the Tauberian theorem quoted in 2] p. 10 give

$$
\lim _{q \rightarrow \infty} q \mathbb{E}_{0}\left[e^{-q \hat{T}_{1}}\right]=\kappa
$$

for some possibly vanishing, explicit constant $\kappa$. This yields

$$
\hat{\mathcal{R}} f(x)=\lim _{q \rightarrow \infty}\left(q^{2} \mathbf{U}_{q} f(x)-q f(x)\right)+\frac{\kappa f(0)}{x^{\alpha}}
$$

and it remains to identify the limit on the right-hand side. Decomposing and changing the variable, one obtains

$q^{2} \mathbf{U}_{q} f_{M}(x)-q f_{M}(x)=q^{2} \int_{0}^{\infty} e^{-q t} \mathbb{E}_{x}\left[f_{M}\left(Z_{t}\right)\right] d t-q f(x)-\int_{0}^{\infty} t e^{-t}\left(\frac{\mathbb{E}_{x}\left[f_{M}\left(Z_{t / q}\right) \mathbf{1}_{\{T \leq t / q\}}\right]}{t / q}\right) d t$.

By the same discussion as above, the Markov property at time $T$ and the a.s. right-continuity of $t \mapsto Z_{t}$ at zero one has, recalling $f_{M}(x)=f(0)$ for every $-M<x<0$,

$$
\begin{aligned}
& \frac{\kappa f(0)}{x^{\alpha}}-\varepsilon(M) \leq \liminf _{q \rightarrow+\infty} \frac{\mathbb{E}_{x}\left[f_{M}\left(Z_{t / q}\right) \mathbf{1}_{\{T \leq t / q\}}\right]}{t / q} \\
& \quad \leq \limsup _{q \rightarrow+\infty} \frac{\mathbb{E}_{x}\left[f_{M}\left(Z_{t / q}\right) \mathbf{1}_{\{T \leq t / q\}}\right]}{t / q} \leq \frac{\kappa f(0)}{x^{\alpha}}+\varepsilon(M)
\end{aligned}
$$

for every $t>0$ and the same constant $\kappa$ as above, with $\varepsilon(M) \rightarrow 0$ as $M \rightarrow \infty$. By Fatou's theorem, this entails

$$
\begin{aligned}
& \frac{\kappa f(0)}{x^{\alpha}}-\varepsilon(M) \leq \liminf _{q \rightarrow+\infty} \int_{0}^{\infty} t e^{-t}\left(\frac{\mathbb{E}_{x}\left[f_{M}\left(Z_{t / q}\right) \mathbf{1}_{\{T \leq t / q\}}\right]}{t / q}\right) d t \\
& \quad \leq \limsup _{q \rightarrow+\infty} \int_{0}^{\infty} t e^{-t}\left(\frac{\mathbb{E}_{x}\left[f_{M}\left(Z_{t / q}\right) \mathbf{1}_{\{T \leq t / q\}}\right]}{t / q}\right) d t \leq \frac{\kappa f(0)}{x^{\alpha}}+\varepsilon(M) .
\end{aligned}
$$

One the other hand, again from the resolvent equation,

$$
q^{2} \int_{0}^{\infty} e^{-q t} \mathbb{E}_{x}\left[f_{M}\left(Z_{t}\right)\right] d t-q f(x)=q^{2} \int_{0}^{\infty} e^{-q t} \mathbb{E}_{x}\left[f_{M}\left(Z_{t}\right)\right] d t-q f_{M}(x) \rightarrow \mathcal{L} f_{M}(x)
$$

as $q \rightarrow+\infty$, where $\mathcal{L}$ is the infinitesimal generator of $Z$. Indeed, one has $f_{M} \in \mathcal{C}_{b}^{2}(\mathbb{R})$ except possibly at zero where its left and right second derivatives are bounded and $f_{M}(x) \rightarrow 0$ as $|x| \rightarrow+\infty$, so that $f_{M} \in \mathcal{D}(\mathcal{L})$, as can be ssen readily from the proof of Theorem 31.5 in [26]. Supposing first $1<\alpha<2$, one has from the Lévy-Khintchine formula

$$
\begin{aligned}
\mathcal{L} f_{M}(x)= & \int_{\mathbb{R}}\left(f_{M}(x+y)-f_{M}(x)-y f_{M}^{\prime}(x)\right) \nu(y) d y \\
= & \int_{0}^{x}\left(f(x-y)-f(x)+y f^{\prime}(x)\right) \frac{c_{-}}{y^{\alpha+1}} d y+\frac{c_{-}}{\alpha x^{\alpha}}(f(0)-f(x))+\frac{c_{-} f^{\prime}(x)}{(\alpha-1) x^{\alpha-1}} \\
& \quad+\int_{0}^{\infty}\left(f(x+y)-f(x)-y f^{\prime}(x)\right) \frac{c_{+}}{y^{\alpha+1}} d y+\varepsilon(M) .
\end{aligned}
$$


Letting $M \rightarrow+\infty$, putting everything together and using the change of variable mentioned in [1] involving the assumption $f \in \mathcal{D}$, one obtains

$$
\hat{\mathcal{R}} f(x)=\mathcal{L} f(x)=\Gamma(-\alpha)\left(c_{-} D_{+}^{\alpha} f(x)+c_{+} D_{-}^{\alpha} f(x)\right)+\frac{c_{-} f(0)}{\alpha x^{\alpha}}
$$

as desired. The cases $\alpha=1$ and $0<\alpha<1$ are analogous and left to the reader.

Remarks 3.2. (a) The above constant $\kappa$ can be identified as $c_{+} / \alpha$, see Lemma 3.1 in [5] and the references therein. The value of this constant does not play any rôle here, but it is interesting to note that it is exactly the same as the one extracted from the Lévy-Khintchine formula in the above proof.

(b) As in the proof of Proposition 2.1, it is possible to relax the condition $f \in \mathcal{D}$. For example when $\alpha \in(1,2)$ the global boundedness condition on $f^{\prime \prime}$ can be changed into $\left(x^{\gamma} \wedge 1\right) f^{\prime \prime}(x)$ bounded on $(0,+\infty)$ for some $\gamma<2-\alpha$, and when $\alpha \in(0,1)$ the global boundedness condition on $f^{\prime}$ can be changed into $\left(x^{\gamma} \wedge 1\right) f^{\prime}(x)$ bounded on $(0,+\infty)$ for some $\gamma<1-\alpha$. This is readily seen from the Lévy-Khintchine formula and the proof of Theorem 31.5 in [26].

(c) With recurrent extensions, it is also possible to give an alternative proof to Proposition 2.2. Suppose as in Section 2 that $\alpha \in(1,2)$ and that $Z$ has no positive jumps. By (3.2), a function is in $\mathcal{D}(\hat{L})$ iff it can be written

$$
\hat{U}_{1} f(x)=\mathbf{U}_{1} f(x)+\mathbb{E}_{x}\left[e^{-T}\right] \hat{U}_{1} f(0)
$$

for some $f \in \mathcal{C}_{0}$. By self-similarity and Formula (7) in [28] - see also the references therein, we find first

$$
\mathbb{E}_{x}\left[e^{-T}\right]=\mathbb{E}_{0}\left[e^{-x^{\alpha} \hat{T}_{1}}\right]=F_{\alpha}(x)-F_{\alpha}^{\prime}(x) .
$$

The term $\mathbf{U}_{1} f(x)$ can be handled with Suprun's formula. Specifically, letting $a \rightarrow+\infty$ in Theorem 1 of [3] and using the discussion made after Theorem 2 therein, we obtain

$$
\begin{aligned}
\mathbf{U}_{1} f(x) & =\left(\int_{0}^{\infty} e^{-y} f(y) d y\right) F_{\alpha}^{\prime}(x)-\int_{0}^{x} F_{\alpha}^{\prime}(y) f(x-y) d y \\
& =\lambda_{f} F_{\alpha}^{\prime}(x)-F_{\alpha}^{\prime} \star f(x)
\end{aligned}
$$

with the notation of Proposition 2.2. Last, we compute

$$
\hat{U}_{1} f(0)=\mathbb{E}\left[\int_{0}^{\infty} e^{-t} f\left(Z_{t}-I_{t}\right) d t\right]=\mathbb{E}\left[\int_{0}^{\infty} e^{-t} f\left(S_{t}\right) d t\right]=\lambda_{f}
$$

where the last equality follows from the discussion after (2.4), paying here attention to the normalizing constants. Putting everything together yields the expression for $\mathcal{D}(\hat{L})$ given in Proposition 2.2. The formula for $\mathcal{D}(L)$ follows the same way, letting $a, x, y \rightarrow+\infty$ with $a-x$ and $a-y$ constant in Theorem 1 of [3] and identifying

$$
\mathbb{E}\left[\int_{0}^{\infty} e^{-t} f\left(\hat{S}_{t}\right) d t\right]=\int_{0}^{\infty}\left(F_{\alpha}^{\prime \prime}(y)-F_{\alpha}^{\prime}(y)\right) f(y) d y .
$$

We omit the details. 
3.2. Second proof of the theorem. In this paragraph we obtain a new proof of the identity (3.1) which does not depend on the results of [28] but on Mellin inversion. More precisely, we will show that

$$
\mathbb{E}_{0}\left[X_{1}^{s}\right]=\mathbb{E}\left[V_{\alpha}^{s}\right] \times \hat{\mathbb{E}}_{0}\left[\hat{X}_{1}^{s}\right]
$$

for every $s \in(1-\alpha, \alpha)$, which is plainly enough to get (3.1). We start with the fractional moments of the random variable $V_{\alpha}$, a computation that could have been made directly by the residue theorem but since most of the argument was already carried out in [29] for some other purposes, we take the opportunity to shorten the proof.

Lemma 3.3. For every $s \in(1-\alpha, \alpha)$, one has

$$
\mathbb{E}\left[V_{\alpha}^{s}\right]=\frac{\sin (\pi / \alpha) \sin (\pi s)}{\alpha \sin (\pi s / \alpha) \sin (\pi(1-s) / \alpha)} .
$$

Proof. By equation (3) in [28] we know that the function

$$
f_{\alpha}(t)=\frac{(-\sin \pi \alpha) t^{\alpha-1}(1+t)}{\pi\left(t^{2 \alpha}-2 t^{\alpha} \cos \pi \alpha+1\right)}
$$

is a probability density over $\mathbb{R}^{+}$. The fractional moments of the corresponding random variable $Y_{\alpha}$ can be computed with the help of the beginning of the proof of Proposition 4 in [29] and a change of variable: one finds

$$
\mathbb{E}\left[Y_{\alpha}^{s}\right]=\frac{\sin (\pi / \alpha) \sin (\pi s)}{\alpha \sin (\pi s / \alpha) \sin (\pi(1+s) / \alpha)}
$$

for every $s \in(-\alpha, \alpha-1)$. Notice that making $s=-1$ entails

$$
\int_{0}^{\infty} v_{\alpha}(t) d t=\mathbb{E}\left[Y_{\alpha}^{-1}\right]=1
$$

which shows that $v_{\alpha}$ is a probability density with an argument slightly different from the introduction. Finally, the fractional moments of $V_{\alpha}$ are given by

$$
\mathbb{E}\left[V_{\alpha}^{s}\right]=\mathbb{E}\left[Y_{\alpha}^{s-1}\right]=\frac{\sin (\pi / \alpha) \sin (\pi s)}{\alpha \sin (\pi s / \alpha) \sin (\pi(1-s) / \alpha)}
$$

for every $s \in(1-\alpha, \alpha)$.

To compute the fractional moments of $X_{1}$ under $\mathbb{P}_{0}$, we will need more material on recurrent extensions and exponential functional of Lévy processes. With the notations of Section 2, let $\hat{Z}=-Z$ be the dual process and $\hat{\mathbb{P}}_{x}$ its law starting at $x>0$. Introducing the stopping time $\hat{T}=\inf \left\{s>0, \hat{Z}_{s}<0\right\}$, consider the positive $(1 / \alpha)$-self-similar Feller process

$$
\hat{R}_{t}=\hat{Z}_{t} \mathbf{1}_{\{\hat{T}>t\}}
$$

The well-known Lamperti transformation [16] shows that the process defined $\xi_{t}=\log \hat{R}_{\tau_{t}}$ for every $t<\hat{T}$, with the notation

$$
\tau_{t}=\inf \left\{s>0, \int_{0}^{s} \hat{R}_{r}^{-\alpha} d r>t\right\},
$$


is a Lévy process starting at $\log x$. Its Lévy-Khintchine exponent $\psi$ which is defined by

$$
\mathbb{E}\left[e^{-\lambda \hat{\xi}_{t}}\right]=e^{t \psi(\lambda)}
$$

for every $\lambda \geq 0$ - recall that $\xi$ has no negative jumps so that the above expectation is finite, has been computed in [5] in terms of a certain improper integral. The next lemma gives a more tractable formulation in terms of Gamma functions.

Lemma 3.4. With the normalization of Section 2, one has $\psi(\lambda)=\Gamma(\lambda+\alpha) / \Gamma(\lambda)$.

Proof. By Corollary 1 in [5] and Theorem 2.4 of [19], one has

$$
\begin{aligned}
\psi(\lambda)=\Phi(\mathrm{i} \lambda) & =\frac{\lambda}{(\alpha-1) \Gamma(-\alpha)}+\int_{0}^{+\infty}\left(e^{-\lambda y}-1+\lambda\left(e^{y}-1\right) \mathbf{1}_{\left\{e^{y} \leq 2\right\}}\right) \frac{e^{y} d y}{\Gamma(-\alpha)\left(e^{y}-1\right)^{1+\alpha}} \\
& =\int_{1}^{+\infty}\left(\frac{y^{-\lambda}-1+\lambda(y-1)}{\Gamma(-\alpha)(y-1)^{1+\alpha}}\right) d y \\
& =\int_{0}^{1}\left(\frac{\left(y^{\lambda}-1\right) y^{\alpha-1}-\lambda(y-1)}{\Gamma(-\alpha)(1-y)^{1+\alpha}}\right) d y+\lambda \int_{0}^{1}\left(\frac{y^{\alpha-2}-1}{\Gamma(-\alpha)(1-y)^{\alpha}}\right) d y \\
& =\frac{\Gamma(\lambda+\alpha)}{\Gamma(\lambda)}-\frac{\lambda}{(\alpha-1) \Gamma(-\alpha)}+\lambda \int_{0}^{1}\left(\frac{y^{\alpha-2}-1}{\Gamma(-\alpha)(1-y)^{\alpha}}\right) d y
\end{aligned}
$$

where we have used several changes of variable. The last integral can be computed with the help of Formula (2.3) in [19]: one gets

$$
\int_{0}^{1}\left(\frac{y^{\alpha-2}-1}{(1-y)^{\alpha}}\right) d y=-\Gamma(1-\alpha)(2-\alpha)_{\alpha}=1 /(\alpha-1)
$$

yielding the desired formula for $\psi$.

Remark 3.5. Supposing now that $Z$ has positive jumps with the notations of Paragraph 3.1, an analogous simplification of Corollary 1 in [5] with Theorem 2.4 of [19] shows the following general formula for the Lévy-Khintchine exponent of the Lévy process associated to $\hat{R}$ by the Lamperti transformation:

$$
\psi(\lambda)=\Gamma(-\alpha)\left(c_{-} \Gamma(\lambda+\alpha) / \Gamma(\lambda)+c_{+} \Gamma(1-\lambda) / \Gamma(1-(\alpha+\lambda))\right)
$$

for every $\lambda \in(-\alpha, 1)$. Setting $\theta=\inf \{\lambda>0: \psi(-\lambda)=0\}$, a simple analysis shows then that $\theta=\alpha \rho=\alpha(1-\hat{\rho})$ where $\hat{\rho}$ is the asymmetry coefficient of $\hat{Z}$, which can also be checked in considering the invariant function of $\hat{R}$ - see again Example 3 in [25].

Setting now

$$
\theta_{-}=\inf \{\lambda>0: \psi(-\lambda)=0\}=1<\alpha,
$$

Theorem 2 in [25] shows the existence of a unique recurrent extension for $\hat{R}$ leaving 0 continuously, whose resolvent $\left(U_{q}^{-}\right)_{q \geq 0}$ is characterized by the formula

$$
U_{q}^{-} f(0)=\frac{\alpha q^{-1 / \alpha}}{\Gamma(1-1 / \alpha) \mathbb{E}\left[I_{-}^{1 / \alpha-1}\right]} \int_{0}^{\infty} f(y) y^{\alpha-2} \mathbb{E}\left[e^{-q y^{\alpha} I_{-}}\right] d y
$$

for any positive measurable function $f$, where

$$
I_{-}=\int_{0}^{\infty} e^{\xi_{s}^{-}} d s
$$


and $\xi^{-}$is a spectrally negative Lévy process with Laplace exponent

$$
\psi_{-}(\lambda)=\psi\left(\alpha \lambda-\theta_{-}\right)=\psi(\alpha \lambda-1)=\frac{\Gamma(\alpha(\lambda+1)-1)}{\Gamma(\alpha \lambda-1)} .
$$

As mentioned in Example 3 of [25], this recurrent extension is the process $X$, and with the help of this identification we can now compute the fractional moments of $X_{1}$ :

Proposition 3.6. For any $s \in(1-\alpha, \alpha)$, one has

$$
\mathbb{E}_{0}\left[X_{1}^{s}\right]=\frac{\sin (\pi / \alpha) \sin (\pi s) \Gamma(s+1)}{\alpha \Gamma(s / \alpha+1) \sin (\pi s / \alpha) \sin (\pi(1-s) / \alpha)} .
$$

Proof. Let us first connect the moments of $X_{1}$ under $\mathbb{P}_{0}$ to those of $I_{-}$. Introducing the positive measurable function $p_{s}(t)=t^{s}$ over $\mathbb{R}^{+}$, for every $s \in(1-\alpha, \alpha)$ one has

$$
\begin{aligned}
\mathbb{E}_{0}\left[X_{1}^{s}\right] & =\frac{q^{s / \alpha+1}}{\Gamma(s / \alpha+1)} \int_{0}^{\infty} e^{-q t} t^{s / \alpha} \mathbb{E}_{0}\left[X_{1}^{s}\right] d t \\
& =\frac{q^{s / \alpha+1}}{\Gamma(s / \alpha+1)} \int_{0}^{\infty} e^{-q t} \mathbb{E}_{0}\left[X_{t}^{s}\right] d t=\frac{q^{s / \alpha+1} U_{q}^{-} p_{s}(0)}{\Gamma(s / \alpha+1)}
\end{aligned}
$$

where the second equality comes from self-similarity and the third from the fact that the resolvent of $X$ is $\left(U_{q}^{-}\right)_{q \geq 0}$. From (3.4) and after some simplifications, this entails

$$
\mathbb{E}_{0}\left[X_{1}^{s}\right]=\frac{\Gamma(1+(s-1) / \alpha) \mathbb{E}\left[I_{-}^{-(1+(s-1) / \alpha)}\right]}{\Gamma(1-1 / \alpha) \Gamma(s / \alpha+1) \mathbb{E}\left[I_{-}^{1 / \alpha-1}\right]}
$$

for any $s \in(1-\alpha, \alpha)$. On the other hand, from Theorem 2.1 and Formula (2.1) in [21] - with our notation which entails $\gamma=1 / \alpha$ therein, see 22 for details - we know that the density function $f_{-}$of $I_{-}$has the alternate series representation

$$
f_{-}(t)=C_{\alpha} \sum_{n=0}^{\infty}(-1)^{n}\left(\frac{\Gamma(n+1+1 / \alpha)}{\Gamma(\alpha(n+1))}\right) t^{-n+1+1 / \alpha}, \quad t>0,
$$

where $C_{\alpha}$ is a positive constant to be determined below. This representation of the density prevents from computing the fractional moments of $I_{-}$by direct integration. Instead, one can use a so-called Mellin-Barnes integral representation of $f_{-}$, which is obtained simply after a contour integration along a big half-circle in the half-plane $x>-1-$ see e.g. Section 3.4 in [13] for details. For any $c \in(-1,0)$ one has

$$
\begin{aligned}
f_{-}(t) & =\frac{C_{\alpha}}{2 \pi \mathrm{i}} \int_{c-\mathrm{i} \infty}^{c+\mathrm{i} \infty}\left(\frac{\Gamma(s+1+1 / \alpha) \Gamma(s+1) \Gamma(-s)}{\Gamma(\alpha(s+1))}\right) t^{-s+1+1 / \alpha} d s \\
& =\frac{C_{\alpha}}{2 \pi \mathrm{i}} \int_{c^{\prime}-\mathrm{i} \infty}^{c^{\prime}+\mathrm{i} \infty}\left(\frac{\Gamma(s) \Gamma(s-1 / \alpha) \Gamma(-s+1+1 / \alpha)}{\Gamma(\alpha s-1)}\right) t^{-s} d s
\end{aligned}
$$

after a change of variable and taking any $c^{\prime} \in(1 / \alpha, 1+1 / \alpha)$. The inversion formula for the Mellin transform - see e.g. formula (3.1.5) in [13] - entails then

$$
\mathbb{E}\left[I_{-}^{s}\right]=C_{\alpha} \frac{\Gamma(s+1) \Gamma(s+1-1 / \alpha) \Gamma(-s+1 / \alpha)}{\Gamma(\alpha(s+1)-1)}
$$


for every $s \in(-1+1 / \alpha, 1 / \alpha)$. Notice in passing, though we shall not need this, that making $s=0$ allows also to compute $C_{\alpha}=\Gamma(\alpha-1) /(\Gamma(1-1 / \alpha) \Gamma(1 / \alpha))$. After some simple transformations, we finally deduce that for any $s \in(1-\alpha, \alpha)$,

$$
\mathbb{E}_{0}\left[X_{1}^{s}\right]=\frac{\Gamma(1+(s-1) / \alpha) \Gamma((1-s) / \alpha) \Gamma(-s / \alpha)}{\alpha \Gamma(1-1 / \alpha) \Gamma(1 / \alpha) \Gamma(-s)}=\frac{\sin (\pi / \alpha) \sin (\pi s) \Gamma(s+1)}{\alpha \Gamma(s / \alpha+1) \sin (\pi s / \alpha) \sin (\pi(1-s) / \alpha)} .
$$

End of the proof. The property that the law of $\hat{X}_{1}$ under $\hat{\mathbb{P}}_{0}$ is that of $S_{1} \stackrel{\mathrm{d}}{=} T_{1}^{-1 / \alpha}$ under $\mathbb{P}$ and a well-known, aforementioned moment formula for $T_{1}$ entails

$$
\hat{\mathbb{E}}_{0}\left[\hat{X}_{1}^{s}\right]=\frac{\Gamma(s+1)}{\Gamma(s / \alpha+1)}
$$

for every $s>-1$, so that (3.3) simply follows from Lemma 3.3 and Proposition 3.6 .

Acknowledgements. The research of PP was supported by a grant from the National Bank of Belgium. TS wishes to thank Grant ANR-09-BLAN-0084-01.

\section{REFERENCES}

[1] V. Bernyk, R. C. Dalang and G. Peskir. Predicting the ultimate supremum of a stable Lévy process with no negative jumps. Preprint, Manchester, 2010. Available at http://arxiv.org/abs/1004.2133

[2] J. Bertoin. Lévy Processes. Cambridge University Press, Cambridge, 1996.

[3] J. Bertoin. Exponential decay and ergodicity of completely asymmetric Lévy processes in a finite interval Ann. Appl. Probab. 7 (1), 156-169, 1997.

[4] R. M. Blumenthal and R. K. Getoor. Markov processes and potential theory. Academic Press, New York, 1968.

[5] M. E. Caballero and L. Chaumont. Conditioned stable Lévy processes and the Lamperti representation. J. Appl. Probab. 43 (4), 967-983, 2006.

[6] P. Carmona, F. Petit and M. Yor. Sur les fonctionnelles exponentielles de certains processus de Lévy. Stoch. Stoch. Rep. 47 (1-2), 71-101, 1994.

[7] P. Carmona, F. Petit and M. Yor. Beta-gamma random variables and intertwining relations between certain Markov processes. Rev. Mat. Iberoamericana 14 (2), 311-368, 1998.

[8] L. Chaumont and M. Yor. Exercises in probability. Cambridge University Press, Cambridge, 2003.

[9] A. ERdelyi. Higher transcendental functions Vol. III. McGraw-Hill, New York, 1953.

[10] P. J. Fitzsimmons. On the existence of recurrent extensions of self-similar Markov processes. Elec. Comm. Probab. 11, 230-241, 2006.

[11] R. Gorenflo, J. Loutchko and Y. Luchko. Computation of the Mittag-Leffler function $E_{\alpha, \beta}(z)$ and its derivatives. Fract. Calc. Appl. Anal. 5, 491-518, 2002.

[12] F. Hirsch and M. Yor. Fractional intertwinings between two Markov semigroups. Potential Anal. 31 (2), 133-146, 2009.

[13] D. Kaminski and R. B. PARIs. Asymptotics and Mellin-Barnes integrals. Cambridge University Press, Cambridge, 2001.

[14] A. A. Kilbas, H. M. Srivastava and J. J. Trujillo. Theory and applications of fractional differential equations. North-Holland, Amsterdam, 2006.

[15] T. J. Kozubowski. Exponential mixture representation of geometric stable distributions. Ann. Inst. Statist. Math. 52 (2), 231-238, 2000.

[16] J. Lamperti. Semi-Stable Markov Processes. I. Z. Wahrsch. verw. Geb. 22, 205-225, 1972.

[17] K. S. Miller and S. G. SAmko. Completely monotonic functions. Integral Transforms Spec. Funct. 12 (4), 389-402, 2001.

[18] M. A. Mourou. Transmutation operators associated with a Dunkl type differential-difference operator on the real line and certain of their applications. Integral Transforms Spec. Funct. 12 (1), 77-88, 2001. 
[19] P. PATiE. Exponential functional of one-sided Lévy processes and self-similar continuous state branching processes with immigration. Bull. Sci. Math. 133 (4), 355-382, 2009.

[20] P. PAtie. Infinite divisibility of solutions to some self-similar integro-differential equations and exponential functionals of Lévy processes. Ann. Inst. H. Poincaré Probab. Statist. 45 (3), 667-684, 2009.

[21] P. PAtie. Law of the exponential functional of one-sided Lévy processes and Asian options. C. R. Acad. Sci. Paris, Ser. I 347, 407-411, 2009.

[22] P. PATiE. Law of the absorption time of positive self-similar markov processes. Preprint, Bern, 2009. Available at http://arxiv.org/abs/0911.1203

[23] M. R. Pistorius. On exit and ergodicity of the spectrally one-sided Lévy process reflected at its infimum. J. Theoret. Probab. 17 (1), 183-220, 2004.

[24] D. Revuz and M. Yor. Continuous martingales and Brownian motion. 3rd edition. Springer, Berlin, 1998.

[25] V. Rivero. Recurrent extensions of self-similar Markov processes and Cramér's condition. Bernoulli 11 (3), 471-509, 2005.

[26] K. Sato. Lévy processes and infinitely divisible distributions. Cambridge University Press, Cambridge, 1999.

[27] W. R. Schneider. Completely monotone generalized Mittag-Leffler functions. Expo. Math. 14 (1), 3-16, 1996.

[28] T. Simon. Fonctions de Mittag-Leffler et processus de Lévy stables sans sauts négatifs. Expo. Math. 28 (3), 290-298, 2010.

[29] T. Simon. Hitting densities for spectrally positive stable processes. Preprint Lille 1, 2010. Available at http://arxiv.org/abs/1002.1540

[30] K. YosidA. Functional Analysis. Springer, Berlin, 1965.

Département de Mathématiques, Université Libre de Bruxelles, Boulevard du Triomphe, B-1050

BRUXELles. Email: ppatie@ac.ulb.be

Laboratoire Paul Painlevé, U. F. R. de Mathématiques, Université de Lille 1, F-59655 Villeneuve D'AscQ CEDEX. Email: simon@math.univ-lille1.fr 